\title{
Geometrodynamics of Electromagnetic Fields in the Newman-Penrose Formalism*
}

\author{
GARRY LUDWIG \\ Department of Mathematics, The University of Alberta, Edmonton, Canada
}

Received February 11, 1970

\begin{abstract}
The "already unified" field theory of Rainich, Misner, and Wheeler is rederived in the spin-coefficient formalism of Newman and Penrose. Conditions equivalent to the Rainich algebraic conditions are obtained by classifying the tracefree Ricci tensor according to its principal null directions. The case of a null electromagnetic field is also treated fully. Necessary and sufficient conditions are given for a Riemannian geometry to have an electromagnetic field, null or non-null, as its source.
\end{abstract}

\section{Introduction}

Many attempts have been made to formulate a unified field theory, that is, one which treats both gravitation and electromagnetism as aspects of the geometry of space-time. These attempts usually take the direction of changing the geometry used in Einstein's theory to accommodate the electromagnetic field as well as the gravitational one. However, Rainich, Misner and Wheeler [1-3] have shown that Einstein's theory is "already unified". Given a Riemannian geometry (with metric tensor $g_{\mu v}$, Ricci tensor $R_{\mu v}$ and Ricci scalar $R$ ) they have found the following necessary and sufficient conditions for this geometry to have a non-null electromagnetic field as its source:

$$
\begin{gathered}
R=0, \\
R_{\mu \alpha} R^{\alpha}{ }_{\nu}=1 / 4\left(R_{\tau \beta} R^{\tau \beta}\right) g_{\mu \nu}, \\
R_{\mu \nu} W^{\mu} W^{\nu} \leqq 0 \quad \text { for any time-like vector } W^{\mu}, \\
R_{\tau \beta} R^{\tau \beta} \neq 0, \\
V_{\lambda ; \tau}-V_{\tau ; \lambda}=0,
\end{gathered}
$$

where

$$
V_{\lambda}=\frac{\sqrt{-g} \varepsilon_{\lambda \nu \beta \gamma} R^{\beta \mu ; \nu} R_{\mu}^{\gamma}}{R_{\varrho \kappa} R^{\varrho \kappa}} .
$$

\footnotetext{
* Supported in part by the National Research Council of Canada.
} 
(Here the semicolon denotes covariant differentiation; $\varepsilon_{\lambda \nu \beta \gamma}$ is the Levi-Civita symbol.) Moreover, if these conditions are satisfied the (nonnull) electromagnetic field can be specified up to an arbitrary constant from the given geometry.

In this paper we shall rederive this result, using the Newman-Penrose formalism, and then proceed to investigate the case of a null electromagnetic field. The latter is fundamentally different from the non-null case. Nevertheless, we can again find necessary and sufficient conditions for a given geometry to have a null electromagnetic field as its source. There arise two distinct situations. Only in one of these is the determination of the electromagnetic field essentially unique.

In the next section we explain the notation. Section 3 consists of a brief summary of the Newman-Penrose formalism. In Section 4 we classify the tracefree Ricci tensor, and in Section 5 we apply this classification to give conditions equivalent to Eqs. (1.1)-(1.4). In Section 6 we rederive the differential condition Eq. (1.5) in its spin-coefficient form and in the final section we find differential conditions for the null-field case.

\section{Notation}

Let us briefly explain the notation used in this paper. Tensor indices are given by small Greek letters and run from 1 to 4 . Spinor indices are given by capital Latin letters and run from 1 to 2 . Small Latin suffices refer to dyad components and also run from 1 to 2 . The summation convention applies throughout. Round brackets around suffices denote symmetrization, square brackets denote antisymmetrization. ${ }^{*}$ denotes the dual of a tensor. A bar over a quantity denotes the complex conjugate of that quantity. Complex conjugation of a spinor is indicated by a bar over the letter and a dot over the spinor index. The correspondence between a tensor and its spinor equivalent is denoted by $\leftrightarrow$.

The signature of space-time is taken to be -2 . The sign of the Riemann tensor $R_{\alpha \beta \gamma \delta}$ is specified by the Ricci identity

$$
n_{\beta ;[\delta ; \gamma]}=\frac{1}{2} R_{\beta \gamma \delta}^{\alpha} n_{\alpha} .
$$

The Ricci tensor is defined by

and the Ricci scalar by

$$
R_{\beta \delta}=R_{\beta \alpha \delta}^{\alpha}
$$

$$
R=R_{\alpha}^{\alpha} .
$$

The Einstein field equations are taken to be

$$
R_{\alpha \beta}-\frac{1}{2} g_{\alpha \beta} R=-T_{\alpha \beta}
$$

where $T_{\alpha \beta}$ is the energy-momentum tensor. 


\section{The Newman-Penrose Formalism}

Our results are derived in the Newman-Penrose spin-coefficient formalism $[4,5]$. We give a short description of this formalism in the present section.

With each point in space-time is associated a two-dimensional complex space whose elements are called spinors. A basis $\left\{k_{A}, m_{A}\right\}$ in this space, normalized by $k_{A} m^{A}=1$, induces in the associated space of vectors a null tetrad $\left\{k_{\alpha}, m_{\alpha}, t_{\alpha}, \bar{t}_{\alpha}\right\}$ satisfying $k_{\alpha} m^{\alpha}=-t_{\alpha} \bar{t}^{\alpha}=1$ with all other inner products vanishing.

The spinor equivalent $\nabla_{D} \dot{Z}$ of the covariant derivative $\nabla_{\alpha}$ can be written

$$
\nabla_{D} \dot{Z}=m_{D} \bar{m}_{\dot{Z}} D-m_{D} \bar{k}_{\dot{Z}} \delta-k_{D} \bar{m}_{\dot{Z}} \bar{\delta}+k_{D} \bar{k}_{\dot{Z}} \Delta,
$$

where $D, \delta, \bar{\delta}, \Delta$ are derivatives in the direction of $k_{\alpha}, t_{\alpha}, \bar{t}_{\alpha}, m_{\alpha}$, respectively. The twelve spin-coefficients $\kappa, \sigma, \varrho, \tau, \varepsilon, \beta, \alpha, \gamma, \pi, \mu, \lambda, \nu$ may be defined as dyad components of the directional derivatives of $k_{A}$ and $m_{A}$ [6]. For example,

$$
D k_{A}=\varepsilon k_{A}-\kappa m_{A} .
$$

The spinor equivalent of the Riemann tensor is given by

$$
\begin{aligned}
R_{A \dot{W} B \dot{X} C \dot{Y} D \dot{Z}}= & \Psi_{A B C D} \varepsilon_{\dot{W} \dot{X}} \varepsilon_{\dot{Y} \dot{Z}}+\varepsilon_{A B} \varepsilon_{C D} \bar{\Psi}_{\dot{W} \dot{X} \dot{Y} \dot{Z}} \\
& +\varepsilon_{A B} \varepsilon_{\dot{Y} \dot{Z}} \Phi_{C D \dot{W} \dot{X}}+\varepsilon_{C D} \varepsilon_{\dot{W} \dot{X}} \Phi_{A B \dot{Y} \dot{Z}} \\
& +2 \Lambda\left(\varepsilon_{A C} \varepsilon_{B D} \varepsilon_{\dot{W} \dot{X}} \varepsilon_{\dot{Y} \dot{Z}}+\varepsilon_{A B} \varepsilon_{C D} \varepsilon_{\dot{W} \dot{Z}} \varepsilon_{\dot{X} \dot{Y}}\right),
\end{aligned}
$$

where $\Psi_{A B C D}$ characterizes the Weyl tensor, $\Lambda=R / 24$, and

$$
-2 \Phi_{A B \dot{W} \dot{X}} \leftrightarrow R_{\mu \nu}-1 / 4 g_{\mu \nu} R .
$$

The dyad components of $\Psi_{A B C D}$ are, as usual, denoted by $\Psi_{0}, \ldots, \Psi_{4}$, and those of $\Phi_{A B \dot{W} \dot{X}}$ by $\Phi_{00}, \Phi_{01}, \ldots, \Phi_{22}$, where, for example,

$$
\Psi_{0}=k^{A} k^{B} k^{C} k^{D} \Psi_{A B C D} .
$$

The dyad components of a bivector $F_{\mu \nu}$, where

$$
F_{\mu \nu} \leftrightarrow \varepsilon_{A B} \phi_{\dot{W} \dot{X}}+\varepsilon_{\dot{W} \dot{X}} \phi_{A B},
$$

are denoted by $\phi_{0}, \phi_{1}, \phi_{2}$.

A change of basis in the spin-space from one normalized basis to another,

$$
\begin{aligned}
& k_{A}{ }^{\prime}=a k_{A}+b m_{A} \quad(a d-b c=1), \\
& m_{A}{ }^{\prime}=c k_{A}+d m_{A},
\end{aligned}
$$


induces a change from one normalized null tetrad to another and a transformation of the dyad components of the various objects. For example, under a null rotation about $k_{A}$ :

$\Psi_{4}$ transforms as

$$
\begin{aligned}
k_{A}{ }^{\prime} & =k_{A}, \\
m_{A}{ }^{\prime} & =c k_{A}+m_{A} .
\end{aligned}
$$

$$
\Psi_{4}{ }^{\prime}=c^{4} \Psi_{0}+4 c^{3} \Psi_{1}+6 c^{2} \Psi_{2}+4 c \Psi_{3}+\Psi_{4} .
$$

Many of the transformation laws required here can be found in the literature [7]. Some others are given below.

For a transformation Eq. (3.3) we find

$$
\begin{aligned}
\kappa^{\prime} & =\kappa, \sigma^{\prime}=\bar{c} \kappa+\sigma, \varrho^{\prime}=c \kappa+\varrho, \\
\varepsilon^{\prime} & =c \kappa+\varepsilon, \tau^{\prime}=c \bar{c} \kappa+c \sigma+\bar{c} \varrho+\tau, \\
\beta^{\prime} & =c \bar{c} \kappa+c \sigma+\bar{c} \varepsilon+\beta, \\
D^{\prime} & =D, \delta^{\prime}=\bar{c} D+\delta, \\
\Delta^{\prime} & =c \bar{c} D+c \delta+\bar{c} \bar{\delta}+\Delta .
\end{aligned}
$$

For a transformation

$$
\begin{aligned}
k_{A}{ }^{\prime} & =a k_{A}, \\
m_{A}{ }^{\prime} & =(1 / a) m_{A},
\end{aligned}
$$

which represents a Lorentz transformation in the $k_{\alpha}-m_{\alpha}$ plane and a rotation in the $t_{\alpha}-\bar{t}_{\alpha}$ plane, we find

$$
\begin{aligned}
\kappa^{\prime} & =a^{3} \bar{a} \kappa, \sigma^{\prime}=a^{3} \bar{a}^{-1} \sigma, \varrho^{\prime}=a \bar{a} \varrho, \\
\tau^{\prime} & =a \bar{a}^{-1} \tau, \pi^{\prime}=\bar{a} a^{-1} \pi, \mu^{\prime}=a^{-1} \bar{a}^{-1} \mu, \\
\varepsilon^{\prime} & =a \bar{a} \varepsilon+\bar{a} D a, \beta^{\prime}=a \bar{a}^{-1} \beta+\bar{a}^{-1} \delta a, \\
D^{\prime} & =a \bar{a} D, \delta^{\prime}=a \bar{a}^{-1} \delta, \Delta^{\prime}=a^{-1} \bar{a}^{-1} \Delta .
\end{aligned}
$$

The spin-coefficient form of Maxwell's equations, the "Ricci identities", the full "Bianchi identities" (actually linear combinations thereof) as well as the commutator relations for the differentiation operators are easily found elsewhere $[4,5]$ and will not be repeated here due to their length.

\section{Classification of the Tracefree Ricci Tensor}

In order to classify the tracefree Ricci tensor $S_{\mu \nu}$, defined by Eq. (3.1), consider the expression

$$
J \equiv \Phi_{A B \dot{W} \dot{X}} l^{A} l^{B} \dot{l}^{\dot{W}} \dot{l}^{X}
$$


and ask for what spinors $l^{A}$ it vanishes. We shall call the corresponding vectors $l_{\alpha}$ principal null vectors of $S_{\mu v}$. These are clearly defined up to a constant only. In terms of dyad components the expression $J$ may be written

where

$$
\begin{aligned}
J= & \Phi_{a b \dot{w} \dot{x}} l^{a} l^{b} \bar{l}^{\dot{w}} \dot{l}^{\dot{x}} \\
= & {\left[\Phi_{00} c^{2} \bar{c}^{2}+2 \Phi_{01} c^{2} \bar{c}+\Phi_{02} c^{2}+2 \Phi_{10} c \bar{c}^{2}+4 \Phi_{11} c \bar{c}\right.} \\
& \left.+2 \Phi_{12} c+\Phi_{20} \bar{c}^{2}+2 \Phi_{21} \bar{c}+\Phi_{22}\right] l^{2} l^{2} \bar{l}^{2} \bar{l}^{\dot{2}},
\end{aligned}
$$

$$
c=\frac{l^{1}}{l^{2}} .
$$

$S_{\mu \nu}$ may belong to one of the following four types ${ }^{1}$.

Type I:

There exists no spinor which will make the expression $J$ vanish. Type II:

There is one non-repeated root $n_{A}$. We find

$$
\begin{aligned}
J & =\left(n_{1} c+n_{2}\right)\left(\bar{n}_{1} \bar{c}+\bar{n}_{2}\right)(C c \bar{c}+D c+\bar{D} \bar{c}+E) \quad(C \text { and } E \text { real }) \\
& =n_{c} l^{c} \bar{n}_{\dot{a}} \bar{l}^{\dot{a}} \bar{\Psi}_{d \dot{b}} l^{d} l^{\dot{b}}
\end{aligned}
$$

that is, $\Phi_{A B \dot{W} \dot{X}}$ is of the form

$$
\Phi_{A B \dot{W} \dot{X}}=n_{(A} \Psi_{B)(\dot{W}} \bar{n}_{\dot{X})} .
$$

Taking $n_{A}$ as basis spinor $k_{A}$ we readily find that

$$
\Phi_{00}=\Phi_{01}=\Phi_{02}=0 .
$$

Conversely, given $S_{\mu v}$ we can find a tetrad in which Eq. (4.1) holds, if and only if the equation $J=0$ has at least one root. This assertion is easily verified by an examination of the transformation equations for $\Phi_{00}, \Phi_{01}, \Phi_{02}$.

Type III:

$S_{\mu \nu}$ has two different principal null directions which, after a suitable normalization, we take as basis in spin-space. Then

$$
\Phi_{A B \dot{W} \dot{X}}=4 \Phi_{11} k_{(A} m_{B)} \bar{k}_{(\dot{W}} \bar{m}_{\dot{X})} ;
$$

all dyad components of $\Phi_{A B \dot{B} \dot{X}}$ except $\Phi_{11}$ vanish. Again, it is easily shown that it is possible to find a basis in which $\Phi_{11}$ is the only non-zero component of $\Phi_{A B \dot{W} \dot{X}}$ if and only if the equation $J=0$ has two distinct roots. It should be noted that the principal null directions determine the spin-space basis only up to a transformation Eq. (3.4). However, $\Phi_{11}$ remains invariant under such a transformation.

\footnotetext{
${ }^{1}$ See note added in proof.
} 
We can distinguish further between Type III a and Type III b. For Type III a, $\Phi_{11}>0$ and $\Phi_{A B \dot{W} \dot{X}}$ is of the form

$$
\Phi_{A B \dot{W} \dot{X}}=\phi_{A B} \bar{\phi}_{\dot{W} \dot{X}},
$$

for Type III b, $\Phi_{11}<0$ and $\Phi_{A B \dot{W} \dot{X}}$ has the form

$$
\Phi_{A B \dot{W} \dot{X}}=-\phi_{A B} \bar{\phi}_{\dot{W} \dot{X}},
$$

where $\phi_{A B}$ corresponds to a non-null bivector as in Eq. (3.2).

Type IV:

$S_{\mu \nu}$ has one repeated principal null direction which we take to correspond to the basis spinor $k_{A}$. Then

$$
\Phi_{A B \dot{W} \dot{X}}=\Phi_{22} k_{A} k_{B} \bar{k}_{\dot{W}} \bar{k}_{\dot{X}} ;
$$

all dyad components other than $\Phi_{22}$ vanish. Conversely, a basis in which all components of $\Phi_{A B \dot{W} \dot{X}}$ except $\Phi_{22}$ vanish can be found if and only if the equation $J=0$ has a repeated root. The principal null direction does not determine the basis uniquely. Transformations with Eqs. (3.3) and (3.4) are still allowed. The former leave $\Phi_{22}$ unchanged, the latter leave $\Phi_{22}$ real with its sign unchanged.

We distinguish between Types IV a and IV b. For the former, $\Phi_{22}>0$ and $\Phi_{A B \dot{W} \dot{X}}$ is of the form Eq. (4.3). For the latter, $\Phi_{22}<0$ and $\Phi_{A B \dot{W} \dot{X}}$ has the form Eq. (4.4), where, in both cases, $\phi_{A B}$ corresponds to a null bivector.

\section{The Algebraic Conditions}

The energy-momentum tensor $T_{\alpha \beta}$ corresponding to an electromagnetic field $F_{\alpha \beta}$ is given by

$$
T_{\alpha \beta}=F_{\alpha}{ }^{\gamma} F_{\gamma \beta}+1 / 4 g_{\alpha \beta} F_{\mu \nu} F^{\mu \nu} .
$$

Since $T_{\alpha}^{\alpha}=0$ the Einstein field equations reduce to

$$
-R_{\alpha \beta}=F_{\alpha}^{\gamma} F_{\gamma \beta}+1 / 4 g_{\alpha \beta} F_{\mu \nu} F^{\mu \nu} .
$$

Translating Eq. (5.1) into spinor language we get Eq. (4.3) with $\phi_{A B}$ related to $F_{\alpha \beta}$ by Eq. (3.2). Therefore, if a geometry has an electromagnetic field $F_{\mu \nu}$ as its source its Ricci tensor is necessarily tracefree and of Type III a or IV a (depending on whether $F_{\mu \nu}$ is non-null or null).

Let us show that these conditions are also sufficient for the existence of a bivector related to $R_{\mu \nu}$ by Eq. (5.1). Suppose first that the Ricci tensor is tracefree and of Type III a. Then

$$
\Phi_{A B \dot{W} \dot{X}} \leftrightarrow-\frac{1}{2} R_{\mu v}
$$


and Eq. (4.2) holds with $\Phi_{11}>0$. If we define

and

$$
\phi_{1}{ }^{\prime}=\sqrt{\Phi_{11}}
$$

$$
\phi_{1}=\phi_{1}{ }^{\prime} \mathrm{e}^{-i \theta}
$$

then the non-null bivector $F_{\mu \nu}$ corresponding to

$$
\phi_{A B}=-2 \phi_{1} k_{(A} m_{B)}
$$

satisfies Eq. (5.1) for any real value of $\theta$, thus proving our assertion for the non-null case.

$\theta$ is the complexion and $F_{\mu \nu}{ }^{\prime}$ obtained from Eq. (5.3) with $\phi_{1}{ }^{\prime}$ in place of $\phi_{1}$ is called the extremal field. The bivectors $F_{\mu \nu}$ and $F_{\mu \nu}{ }^{\prime}$ are related by a duality rotation:

$$
\begin{aligned}
& F_{\mu \nu}=\cos \theta F_{\mu \nu}{ }^{\prime}+\sin \theta F_{\mu \nu}^{{ }^{\prime}{ }^{\prime},}, \\
& F_{\mu \nu}^{*}=-\sin \theta F_{\mu \nu}{ }^{\prime}+\cos \theta F_{\mu \nu}{ }^{* \prime} .
\end{aligned}
$$

Next, suppose the Ricci tensor is tracefree and of Type IVa. Then Eq. (5.2) holds and $\Phi_{A B \dot{W} \dot{X}}$ is given by Eq. (4.5) with $\Phi_{22}>0$. Proceeding as with the non-null case we define

and

$$
\phi_{2}{ }^{\prime}=\sqrt{\Phi_{22}}
$$

$$
\phi_{2}=\phi_{2}{ }^{\prime} e^{-\imath \hat{\theta}} \quad(\theta \text { real }) .
$$

The null bivector corresponding to

$$
\phi_{A B}=\phi_{2} k_{A} k_{B}
$$

satisfies Eq. (5.1) for any real value of $\theta$, thus proving our assertion for the null case as well.

The conditions we have just found are equivalent to Eqs. (1.1)-(1.3) (with Eq. (1.4) valid for the non-null case and $R_{\mu \nu} R^{\mu \nu}=0$ for the null case). It is easy to show that

where

$$
S_{\alpha}^{\beta} S_{\beta \gamma} \leftrightarrow 4 \psi_{(A C)(\dot{W} \dot{Y})}+\varepsilon_{A C} \varepsilon_{\dot{W} \dot{Y}} \Phi_{B D \dot{X} \dot{Z}} \Phi^{B D \dot{X} \dot{Z}},
$$

$$
\psi_{A C \dot{W} \dot{Y}} \equiv \Phi_{A B \dot{W} \dot{X}} \Phi_{C}^{B} \dot{X} \dot{Y} .
$$

A straightforward but tedious calculation will then show that $\psi_{(A C)(\dot{W} \dot{Y})}$ vanishes if and only if $\Phi_{A B \dot{W} \dot{X}}$ can be written as in Eq. (4.3) or Eq. (4.4). In that case $S_{\mu \nu}$ satisfies Eq. (1.2), which is, therefore, equivalent to the statement that the traceface Ricci tensor belongs to Type III or IV. Assuming now that $S_{\mu v}$ belongs to Type III or IV, we can easily prove that it is of Type III if and only if it obeys Eq. (1.4) and of Type IV otherwise. With the same assumption it is equally straightforward to show that Eq. (1.3) is satisfied if and only if $S_{\mu \nu}$ is of Type IIIa or IVa. 
This proves that the algebraic conditions as stated in this section are equivalent to the ones given usually.

\section{Differential Conditions in the Non-null Case}

The conditions found in the last section are purely algebraic, in the sense that they do not involve the change in any quantities as one moves from world-point to world-point. In the present section we shall derive differential conditions equivalent to Eq. (1.5) (for the non-null case).

Let us first find a necessary condition on the geometry in order that the bivector $F_{\mu \nu}$ corresponding to $\phi_{A B}$ as given by Eq. (5.3) satisfy Maxwell's source-free equations. With our choice of tetrad these equations become [4]

$$
\begin{aligned}
D \phi_{1} & =2 \varrho \phi_{1}, \\
\delta \phi_{1} & =2 \tau \phi_{1}, \\
\bar{\delta} \phi_{1} & =-2 \pi \phi_{1}, \\
\Delta \phi_{1} & =-2 \mu \phi_{1} .
\end{aligned}
$$

Also, those "Bianchi identities" equivalent to $T_{\mu}^{v} ;_{v}=0$ are given by [5]

$$
\begin{aligned}
& D \Phi_{11}=(2 \varrho+2 \bar{\varrho}) \Phi_{11} \\
& \delta \Phi_{11}=(2 \tau-2 \bar{\pi}) \Phi_{11} \\
& \bar{\delta} \Phi_{11}=(2 \bar{\tau}-2 \pi) \Phi_{11} \\
& \Delta \Phi_{11}=-(2 \mu+2 \bar{\mu}) \Phi_{11} .
\end{aligned}
$$

(In this formalism the well-known fact that Maxwell's equations imply the vanishing of $T_{\mu}^{v} ;{ }_{v}$ becomes quite obvious.)

Now,

$$
\begin{aligned}
D \Phi_{11} & =D\left(\phi_{1}{ }^{\prime} \phi_{1}{ }^{\prime}\right) \\
& =2 \phi_{1}{ }^{\prime} D\left(\phi_{1} e^{i \theta}\right) .
\end{aligned}
$$

Using Eqs. (6.1) and (6.2) we obtain

$$
D \theta=i(\varrho-\bar{\varrho}) .
$$

Continuing in this fashion we also find

$$
\begin{aligned}
& \delta \theta=i(\tau+\bar{\pi}), \\
& \bar{\delta} \theta=i(-\bar{\tau}-\pi), \\
& \Delta \theta=i(\bar{\mu}-\mu) .
\end{aligned}
$$

Each of Eqs. (6.3) is unchanged (apart from a multiplicative factor) by a transformation Eq. (3.4). The existence of a solution $\theta$ depends, there- 
fore, only on the geometry. A solution exists if and only if certain compatibility conditions are satisfied. For example, operating with $\delta$ on Eq. (6.3a), with $D$ on Eq. $(6.3 \mathrm{~b})$, subtracting and using one of the commutator relations we obtain an equation depending only on the spin-coefficients and their derivatives. The same holds true for three more conditions found in a similar way. Since these four conditions are independent of the remaining freedom of choice in the tetrad they clearly represent a restriction on the geometry. In fact, a straightforward but tedious calculation shows that Eq. (1.6) and Eqs. (6.3) are equivalent.

Conversely, if the given geometry satisfies the algebraic conditions and is such that Eqs. (6.3) are compatible we can find a solution $\theta$ and define

$$
\phi_{1}=\sqrt{\Phi_{11}} e^{-i \theta}
$$

Reversing the argument given above we can show that the corresponding non-null bivector satisfies not only Eq. (5.1) but also Maxwell's equations. This bivector is almost uniquely determined. For if $\theta$ and $\theta^{\prime}$ are two solutions of Eqs. (6.3), their difference $\theta^{\prime \prime}=\theta-\theta^{\prime}$ must be such that $D \theta^{\prime \prime}=\delta \theta^{\prime \prime}=\bar{\delta} \theta^{\prime \prime}=\Delta \theta^{\prime \prime}=0$, that is, it must be a constant.

In summary, the necessary and sufficient condition for a Riemannian geometry to have as its source a non-null bivector which satisfies Maxwell's equations is that the Ricci tensor be tracefree and of Type III a and that Eqs. (6.3) be compatible.

\section{Differential Conditions in the Null Case}

We now assume that the algebraic conditions for the null case are satisfied and obtain differential conditions which are necessary and sufficient for a Riemannian geometry to have as its source a null bivector which is a solution of the source-free Maxwell's equations. We choose a basis in spin-space for which

$$
-\frac{1}{2} R_{\mu \nu} \leftrightarrow \Phi_{A B \dot{W} \dot{X}}=k_{A} k_{B} \bar{k}_{\dot{W}} \bar{k}_{\dot{X}}
$$

Again, this choice is not unique. Transformations of the type Eq. (3.3) or Eq. (3.4) with $a=e^{i \phi}$ (where $\phi$ is real) leave the form of Eq. (7.1) invariant.

The concepts of extremal field and complexion are no longer independent of the remaining freedom in our choice of tetrad. Nevertheless, for any given choice we define the complexion by

$$
\phi_{A B}=k_{A} k_{B} e^{-i \theta} .
$$

The corresponding bivector is null and related to the Ricci tensor by Eq. (5.1). It is our aim to find conditions on the geometry making it 
possible to determine the complexion $\theta$ in such a way that the bivector defined by Eq. (7.2) satisfies Maxwell's equations. The latter can be written

and, since $\theta$ is real,

$$
\begin{aligned}
\kappa & =\sigma=0, \\
D \theta & =i(2 \varepsilon-\varrho), \\
\delta \theta & =i(2 \beta-\tau),
\end{aligned}
$$

$$
\bar{\delta} \theta=i(\bar{\tau}-2 \bar{\beta}) .
$$

According to Eq. (7.3) the null congruence defined by the principal null direction of the Ricci tensor is necessarily geodesic and shearfree.

A transformation Eq. (3.3) leaves $k_{A}, \theta$ and hence $\phi_{A B}$ invariant. A transformation Eq. (3.4) with $a=e^{i \phi}$ changes

If we demand that

$$
k_{A} \rightarrow e^{i \phi} k_{A} \text {. }
$$

$$
\theta \rightarrow \theta+2 \phi,
$$

$\phi_{A B}$ will again be invariant. Moreover, with the transformation law Eq. (7.5), assuming Eq. (7.3) is valid, Eqs. (7.4) are covariant under the remaining freedom we have in choosing the tetrad. (This can easily be established with the aid of the transformation formulas listed in Section 3 ). Therefore, whether $\theta$ can be determined so that $\phi_{A B}$ (which is independent of the particular choice in tetrad we made) satisfies Maxwell's equations depends solely on the geometry.

Let us consider the compatibility conditions on Eqs. (7.4) assuming Eq. (7.3) to be valid. If the geodesic null congruence defined by the principal null direction of the Ricci tensor is hypersurface orthogonal $(\varrho=\varrho)$, then these compatibility conditions involve only spin-coefficients and their derivatives. If they are satisfied, Eqs. (7.4) can be integrated and a bivector obeying Maxwell's equations can be found. However, the complexion is far from unique; for if $\theta$ and $\theta^{\prime}$ are two solutions their difference $\theta^{\prime \prime}=\theta-\theta^{\prime}$ must satisfy only $D \theta^{\prime \prime}=\delta \theta^{\prime \prime}=\bar{\delta} \theta^{\prime \prime}=0$, with $\Delta \theta^{\prime \prime}$ completely arbitrary. An example of such a geometry is exhibited in Ref. [3].

If the geodesic null congruence is not hypersurface orthogonal, then the compatibility condition for Eqs. (7.4b) and (7.4c) takes on the form

$$
\Delta \theta=\frac{1}{\varrho-\bar{\varrho}}[A] \text {, }
$$

where $[A]$ is an expression involving spin-coefficients and their derivatives. The complexion must now satisfy Eqs. (7.4) and (7.6) and, if these equations are compatible, will be unique (up to a constant) as in the non-null case. 
In summary, in order for a Riemannian geometry to have as its source a null electromagnetic field satisfying Maxwell's source-free equations the Ricci tensor must be tracefree and of Type IVa, and the null congruence associated with its principal null direction must be geodesic and shearfree. If this congruence is hypersurface orthogonal necessary and sufficient conditions are given by the compatibility requirements on Eqs. (7.4); if not, by the compatibility requirements on Eqs. (7.4) and (7.6). Only in the latter case is the complexion determined up to an additive constant.

Note added in proof. The classification of the tracefree Ricci tensor as given in Section 4 is incomplete. Moreover, what was considered to be a non-repeated root of $J=0$ is actually a two-fold root. This becomes clear from the following consideration. It is possible for $J$ to have the form

$$
J=\left(n_{A} p_{B} \bar{q}_{\dot{W}} \bar{r}_{\dot{X}}+q_{A} r_{B} \bar{n}_{\dot{W}} \bar{p}_{\dot{X}}\right) l^{A} l^{B} \bar{l}^{W} \bar{l}^{\dot{X}},
$$

in which case $J$ has four roots, namely $n_{A}, p_{A}, q_{A}$ and $r_{A}$. If $n_{A}$ and $q_{A}$ coincide we get type II, if $n_{A}=q_{A}$ and $p_{A}=r_{A}$ we get type III. More types than the ones listed in Section IV will clearly arise.

This incompleteness of the classification in no way affects the remainder of the paper since all that is required is that types III and IV be separate algebraic classes of the tracefree Ricci tensor, which indeed they are.

\section{References}

1. Rainich, G. Y.: Trans. Am. Math. Soc. 27, 106 (1925).

2. Misner, C. W., Wheeler, J. A.: Ann. Phys. 2, 525 (1957).

3. Witten, L.: A geometric theory of the electromagnetic and gravitational fields, (Gravitation, ed. by L. Witten). New York: Wiley, 1962.

4. Newman, E. T., Penrose, R.: J. Math. Phys. 3, 566 (1962).

5. Pirani, F. A. E.: Lectures on general relativity, (Brandeis Summer Institute, 1964). Englewood Cliffs, N. J.: Prentice Hall 1965.

6. Ludwig, G.: Analogue of the Goldberg-Sachs theorem (to appear in J. Math. Mech.).

7. - Classification of electromagnetic and gravitational fields: Am. J. Phys. 37, 1225 (1969).

G. Ludwig

Department of Mathematics

The University of Alberta

Edmonton 7, Alberta, Canada 